FISSION CROSS-SECTION IN URANIUM AND THORIUM FOR DEUTERON IMPACT

\author{
By J. C. Jacobsen and N. O. Lassen \\ Institute of Theoretical Physics, University of \\ Copenhagen. Mày 8
}

As reported in a previous note ${ }^{1}$, experiments have been carried out with the cyclotron in this institute to investigate the cross-section for fission in thorium and uranium produced by impact of deuterons with different energies. The fission outputs were determined by collecting the radioactive fragments on aluminum foils facing the targets and screened from the deuteron beam. Because of the difficulty of estimating the number of fission processes from the $\beta$-radioactivity of these foils, a considerable uncer. tainty, however, was involved in the determination of the absolute value of the fission cross-section.

In continued experiments, this uncertainty has been reduced by a calibrating procedure in which two thin uranium targets were exposed to neutron impact. One of the targets was placed in a small ionization chamber and the number of fission particles emitted from this target counted by a linear amplifier. The fission particles from the second target were collected on a thin lead foil ; the distance between the two targets was sufficiently small to ensure that the neutron intensity was very nearly the same. By measuring the activity of the collecting foil with the same counting arrangement as in the experiments with deuterons, the ratio between the foil activity and the number of fission particles emitted during the irradiation could thus be determined.

Instead of the preliminary value of $0.5 \times 10^{-26} \mathrm{~cm}^{2}$ given in the previous note, the measurements thus calibrated gave now the value $(2 \cdot 2 \pm \mathrm{I}) \times 10^{-26} \mathrm{~cm} .^{2}$ for the fission cross-section in uranium at $9-\mathrm{Mev}$. deuteron energy. The results found for the variation of the cross-section with deuteron energy and the ratio between the cross-sections in thorium and uranium given in the note remain, of course, unaltered. From the value 0.7 for the last ratio, which agrees with the independent determination by Krishnan and Banks ${ }^{2}$, we obtain in consequence $(1 \cdot 5 \pm 0 \cdot 7) \times 10^{-26} \mathrm{~cm}^{2}$ for the fission cross-section in thorium at $9-\mathrm{Mev}$. deuteron energy.

Details of the experiments are described in a paper in print in the Communications of the Copenhagen Academy of Sciences where, also, a description of the cyclotron has just been published .

${ }^{1}$ Jacobsen, J. C., and Lassen, N. O., Phys. Rev., 58, 867 (1940).

${ }^{2}$ Krishnan, R. S., and Banks, T. E., NATURE, 145, 860 (1940).

J Jacobsen, J. C., Det Kgl. Danske Vidensk. Selsk. Math.-fys. Medd. 19, 2 (1941).

\title{
EDUCATIONAL PROGRESS IN THE UNITED STATES
}

\section{By $R$. WEATHERALL Eton College}

$I_{c}^{N}$ recent years, C. V. Good, of the University of Cincinnati, has made a practice of preparing an annual review of educational progress in the United States $^{1}$. In his report for 1940 his method has been to compile a list of educational issues, and then to cite illustrations of the manner in which they are being met. These issues may be summarized as follows:

\section{Democratic Society and Public Education}

A great deal of thought is being given to the problem of education for good citizenship in a democratic society. To achieve such an aim requires individuals who have respect for other people, a concern for group problems, and a willingness to use intelligence in problem solving. In dealing with educational matters there is a marked tendency towards co-operative effort. This includes the pooling of ideas by teachers and administrators, and extensive use, is being made of joint discussions between teachers and students. In this way the students themselves are being encouraged to ask and answer questions which should help them to evaluate current social, economic and political trends, and to establish helpful relations with other members of the community.

\section{Education and the Emergencies of National DEFENCE}

Almost to a greater extent than in Great Britain, teachers and the general public alike in the United States are giving consideration to the repercussions which the present world crisis is having upon the educational field, and to the extent to which by educational means support may be given to the national effort. As the report points out, education is called upon to defend human resources, culture, and American democratic processes, as well as to assist in the material defence of the community and nation. Federal participation in education is extending, and in the service of youth, unification in Federal policy is being brought about in the spheres of health, education, and social welfare.

\section{Youth Problems and CurRiculum}

Under this heading consideration is being given to the needs of the large number of pupils who will not proceed to college, and who do not require specific technical training. The problem of the responsibility of the community towards young people between the ages of eighteen and twenty-one, who cannot be readily absorbed into industry, is also receiving attention. The United States Employment Service is concerning itself with the adjustment of schoolleavers to suitable occupations, and with the best way of enabling young people to enter upon rural life. Other investigations are dealing with the specific problem of Negro students: their development during adolescence, and their status in the existing social and economic order.

To a far greater extent than occurs in the British Isles, public opinion makes itself directly felt in educational policy, and recourse is being made to the 